anbiances Environnement sensible, architecture et espace urbain

\title{
"Prendre les apparences au pied de la lettre »
}

A propos de : Jocelyn Benoist, Le bruit du sensible, Paris, Editions du Cerf, coll. « Passages », 2013.

Appearances taken literally

\section{Anthony Pecqueux}

\section{OpenEdition}

\section{Journals}

Édition électronique

URL : http://journals.openedition.org/ambiances/475

DOI : 10.4000/ambiances.475

ISSN : 2266-839X

\section{Éditeur :}

Direction Générale des Patrimoines - DAPA - MCC, UMR 1563 - Ambiances Architectures Urbanités $(\mathrm{AAU})$

\section{Référence électronique}

Anthony Pecqueux, « «Prendre les apparences au pied de la lettre » », Ambiances [En ligne], Comptesrendus, mis en ligne le 13 novembre 2014, consulté le 22 septembre 2020. URL : http:// journals.openedition.org/ambiances/475 ; DOI : https://doi.org/10.4000/ambiances.475

Ce document a été généré automatiquement le 22 septembre 2020.

\section{c)}

Ambiances is licensed under a Creative Commons Attribution-NonCommercial-NoDerivatives 4.0 International License. 


\title{
«Prendre les apparences au pied de la lettre»
}

\author{
A propos de : Jocelyn Benoist, Le bruit du sensible, Paris, Editions du \\ Cerf, coll. « Passages », 2013. \\ Appearances taken literally
}

Anthony Pecqueux

\section{RÉFÉRENCE}

Jocelyn Benoist, Le bruit du sensible, Paris, Editions du Cerf, coll. « Passages », 2013.

1 Avec ce nouveau livre, le philosophe Jocelyn Benoist poursuit une voie passionnante qu'il déploie livre après livre ${ }^{1}$, en repoussant à chaque fois - patiemment, et dans un langage accessible et vivant (alors que les démonstrations sont souvent techniques, ardues) - les limites de notre compréhension de la perception et du sensible, qui ont tant intrigué la phénoménologie dont il est issu, et qui constituent une large part de la discussion philosophique actuelle. La thèse centrale de ce livre est la suivante : les sens sont muets ${ }^{2}$ mais le sensible est bruyant. Jocelyn Benoist s'attèle ainsi à opérer en quelque sorte un dépassement de la phénoménologie de l'intérieur, principalement sur la question du lien entre perception et intentionnalité (question primordiale depuis Husserl), en se centrant moins sur la perception que sur le sensible qui permet d'envisager une sortie hors du schéma intentionnel - ce que la focalisation sur la perception ne permet pas. Benoist opère cette mutation (de la perception au sensible) moins par une opposition entre perçu et sensible que par la patiente mise en évidence de leurs différences grammaticales : du vu au visible, du perçu au sensible.

2 Pour cela, il ne se contente pas de reprendre à Austin son argument sur le mutisme des sens, il lui emprunte également une méthode d'investigation : celle qu'Austin dénomme "phénoménologie linguistique » (ou : «ce que nous dirions quand », Austin, 1994, p. 144 ; comme dans le reste de cette note de lecture, c'est l'auteur qui souligne), appelée ici 
« analyse grammaticale » en référence directe bien sûr à la méthode wittgensteinienne. Pour le dire rapidement, cette méthode, qui est plus généralement celle de la philosophie du langage ordinaire, consiste à se départir des problèmes créés de toute pièce par les philosophies traditionnelles pour revenir à «l'homme de la rue » (plain man, Austin, 2007); par exemple : décrire et discuter de la perception à partir de ce qu'en dirait l'homme de la rue (ou à partir du vocabulaire des objets courants, selon l'expression de Gilbert Ryle). Ici, on le verra, la principale cible de Benoist concerne les illusions perceptives qui n'ont presque jamais, dans la vie ordinaire, les effets perturbants que les philosophes s'imaginent.

3 Par là, ce livre part dans une guerre fine contre la façon dont la philosophie de l'esprit et les sciences cognitives se sont accaparées l'approche philosophique de la perception, qui connaît une croissance exponentielle sous leur houlette mais dans une direction univoque : la connaissance que la perception est susceptible de porter. A cela, il oppose d'emblée que la perception n'est ni capacité ni mode de connaissance mais un fait, contre lequel il est incongru de lutter ou qu'il est malaisé de vouloir contourner puisque c'est «l'un des noms mêmes de la réalité » (p. 9). Je vais rendre compte de ce livre en décomposant ce qui me semble être ses cinq principaux arguments: 1/ l'ancrage des concepts ordinaires dans la perception; 2/ la force de l'évidence perceptuelle; 3 / la portée ontologique du visible; 4/ l'appartenance du langage à l'univers sensible ; et $5 /$ la transparence du vu au voir.

$4 \quad 1 /$ Le premier a trait à ce que la philosophie traditionnelle appelle le problème de la perception, comme tentative de saisir les liens entre l'esprit et le monde. Pour Benoist, l'enjeu est de parvenir à montrer qu'il n'y a pas à faire intervenir la moindre «faculté 'supérieure' " (p. 30) pour les saisir. Afin de poser à nouveaux frais ce caractère d'évidence perceptuelle, il utilise une première fois sa méthode grammaticale en l'appliquant à la façon dont les objets nous apparaissent ordinairement (ou: familièrement, habituellement). Cela le conduit à mettre en évidence toute une gamme de concepts qu'il qualifie de "'naturels' » dans la mesure où ils " renvoient aux choses telles qu'elles nous apparaissent familièrement et déterminent leur prise sur elles d'une façon qui est précisément indissociable de ce mode d'apparaître familier » (p. 35); en somme, des concepts ordinaires. Cette argumentation est décisive car elle renverse la question de la philosophie traditionnelle : il n'est plus question d'une perception qui "donnerait » un objet ou un accès au monde, mais d'une perception comme «ce par rapport à quoi seulement la notion d'objet (ou en tout cas une certaine notion ordinaire d'objet) $a$ un sens » (p. 37). Autre façon de le dire : par là, Benoist démine l'habituelle (et stérile) opposition entre concepts et percepts puisque ces derniers fondent nombre des premiers.

5 2/ Le deuxième principal argument de Benoist, situé dans le chapitre 3 sur la « radicalité du perçu », s'appuie sur un examen des dites « illusions perceptuelles » : en sont-elles vraiment? A partir de la célèbre illusion de Müller-Lyer, l'auteur montre que nous percevons des formes (Gestalts) sensibles différentes selon qu'un segment se termine par des flèches rentrées ou sortantes: les deux segments ont beau avoir une longueur identique, ils ne se ressemblent guère. Mieux dit, ils «n'ont pas vraiment le même air » (p. 95). Pour Benoist, des «problèmes » tels que les illusions perceptuelles ne font rien d'autre que montrer la tendance persistante des philosophies traditionnelles de la perception à 
«sous-estimer la force incroyable de l'évidence perceptuelle - force fondée sur l'importance de cette part de nos concepts qui sont tout juste faits pour la perception et qui font d'elle, sous autant d'aspects différents, une preuve » (p. 101).

6 On comprend dès lors l'importance des prémisses argumentatives de l'auteur: ce second argument est déplié grâce aux concepts ordinaires fondés sur la perception et à la méthode grammaticale; bref : grâce à la façon dont les choses nous apparaissent ordinairement et non à la façon dont les philosophes les imaginent dans leur construction de « problèmes ».

7 3/ Le troisième argument, central, prend place dans le chapitre 4 où l'auteur nous fait parcourir le chemin qui le mène à déplacer la problématique du perçu au sensible, du $\mathrm{vu}$ au visible. Pour cela, il affronte des questions cruciales telles que : qu'est-ce que "voir un homme né à Jérusalem » (reprenant ainsi un exemple d'Austin, 2007, p. 188-189) ? Cela complexifie considérablement le problème en posant la question de déterminations non-perceptuelles, liées à un savoir pré-existant ou à un savoir social. Pour étoffer sa démonstration, Benoist part d'un exemple simple : est-ce que je vois une tache blonde ou une étudiante en philosophie? Il introduit alors l'argument du contexte et de la situation, mais surtout de la familiarité pour récuser qu' "étudiante en philosophie » soit une détermination non phénoménologique, non visible (sensible) à l'œil (et/ou un autre organe sensoriel) nu :

«Il est possible, en ce sens, qu'il n'y ait pas de concepts radicalement, 'en soi', non phénoménologiques, mais que tous, en tout cas beaucoup, puissent se voir chargés phénoménologiquement par la fréquentation, l'expérience sensible que nous avons de l'objet » (p. 114).

8 Cette voie permet de poser le couplage, l'appariement entre concept et expérience qui fait que, tout simplement, je vois « mon étudiante » (p. 118-119).

9 Cette analyse poursuit de montrer à quel point nombre de nos concepts sont imprégnés, investis par la perception, sans pour autant que les concepts perceptuels soient prioritaires : le concept «mon étudiante " n'a a priori rien de perceptuel mais a fini par se fondre avec l'apparence de cette étudiante; a contrario, les concepts perceptuels, ceux de couleur notamment (comme «tache blonde »), peuvent fournir de mauvaises réponses localement - dans un contexte. C'est en ce sens que Benoist plaide pour une ontologie de la perception, à savoir non pas pour une nouvelle ontologie ou une ontologie spécifique, mais pour une réflexion qui reconnaisse que « notre concept d'être est profondément imprégné du commerce perceptuel que nous avons avec ce que nous disons être » (p. 123). Ainsi, la perceptibilité apparaît comme une dimension cardinale de l'être, issue du couplage concept / expérience; par exemple, le couple entre « mon étudiante » et l'apparence de cette jeune femme blonde.

Cette poussée de l'argument premier jusque dans ses conséquences ontologiques conduit Benoist à mettre en avant une certaine conception de la réalité ; « en tant que perceptible » (p. 129). Elle implique donc de prendre en compte la force des apparences (physionomies) des choses, du monde. Elle permet également de faire de la visibilité la règle générale, à savoir : " pas de vu sans visibilité » (p. 132). "Ce que nous appelons alors visible, dans sa réalité, c'est tout simplement ce qui rend visible quelque chose qui est vu ». Il donne encore à cette visibilité le joli nom de "pulpe du sensible ", saisie dans la perception sous forme de Gestalt (dont il préconise un usage en tant que notion ontologique et non psychologique) qui opère ainsi l'identification de ce qui est vu. 
11 4/ Passées ces pages décisives, et arrivé à ce stade de son argumentation, Benoist n'a plus qu'à déplier deux conséquences logiques. La première forme son quatrième argument, logé dans le chapitre 6 sur le "bruit du sensible »; elle consiste à affirmer l'«appartenance fondamentale du langage à l'univers sensible» (p. 177) 3 . Sur cette pente, l'auteur nous conduit en compagnie de la "phénoménologie du son" d'Emmanuel Lévinas, pour qui la spécificité du son transparaît dans son aspect retentissant : le sensible y éclate (donc en dehors de toute intentionnalité), alors qu'il a été domestiqué en vue de la vérité à travers le visible. Ce qu'apporte alors centralement le son est de faire apparaître le sensible comme une épreuve non de l'intériorité mais de l'extériorité, à l'encontre de toute une tradition. En outre, selon la conception lévinassienne, le son s'éprouve en quelque sorte en supplément gratuit du sensible, " comme si nous avions plus de sensible qu'il n'en faut pour faire sens de la chose» (p. 199), ce qui ferait d'autant mieux résonner le sensible en lui-même dans la mesure où la chose s'est déjà manifestée par ailleurs.

Pour Benoist, cette analyse permet de penser une voie de sortie du schéma intentionnel classique (pour la phénoménologie et au-delà), dans la mesure où le sensible sonore devient «ce qui se révèle comme le fait une réalité. Ce à quoi nous sommes mis en position de répondre, plus que de le thématiser » (p. 202). Cette spécificité nous ramène aux pages plus connues de Lévinas sur le visage et la voix; du coup, sous cet angle, le son manifesterait "l'immixtion de l'autre (mais pas encore de l'autrui) au niveau phénoménologique même» (p. 203). L'intérêt d'une telle analyse est d'orienter la compréhension du sensible à rebours des questions d'intentionnalité et de vérité (de connaissances, etc.), en direction d'une dimension essentiellement praxéologique : liée à l'autre et à ses activités ${ }^{4}$. Le son n'y représente pas plus qu'un accès privilégié : il ne doit donc pas être déconnecté des autres modalités sensorielles.

5/ La seconde conséquence logique de la règle générale de la visibilité, à savoir le cinquième et dernier argument de l'auteur, forme le chapitre conclusif sur «l'être sensible » et scelle la «transparence du vu au voir » (p. 206) : il n'y a rien de «donné » dans et par la perception, il n'y a rien de plus que l'objet perçu. Thèse finale triviale mais qui modifie radicalement nos manières d'appréhender la perception et le sensible, puisque par là «la grammaire du perçu s'identifie purement et simplement à la grammaire de l'objet» (p. 207). Le sensible s'y trouve alors à sa juste place, ni hégémonique (toujours pas d'ontologie spécifique de la perception à faire valoir pour Benoist) ni subalterne : sa centralité ontologique tient à ce qu'il participe de manière décisive à la façon dont nous formons nos concepts et parmi eux celui d'être (p. 210), par contact direct, perceptuel.

Arrivé précautionneusement à ce stade quasi-tautologique, Benoist opère une bifurcation saisissante en direction des arts : il quitte alors la grammaire de l'objet pour celle du sensible comme ressource. La question ne se limite plus à ce qui est vu, pour s'ouvrir aussi à «ce dont est fait ce qui est vu» (p. 225) : bel et bien le sensible comme ressource ou matériau. Il interprète alors, en suivant l'analyse baudelairienne, l'irruption de la modernité esthétique à partir de Delacroix, comme le passage d'un «faire voir l'objet " (de l'ordre testimonial, d'administration de la preuve) à un « faire voir le visible » (entendre l'audible, etc.) (p. 226sqq). Par exemple, le travail du peintre ne porte plus tant sur l'objet ou sa représentation, que sur la mise en évidence du visible même, donc sur les conditions par lesquelles un objet (une forme) est rendu(e) visible. La même chose vaut sur le plan sonore avec l'introduction de la musique 
atonale, qui fait porter l'enquête (du musicien comme de l'auditeur) sur l'audibilité. Ce que les arts nous apprennent alors, à travers le dévoilement du caractère normatif de la norme, c'est à problématiser le sensible en laissant de côté la perception et donc la compréhension, et ainsi à « libérer le sensible» (p. 236). ontologie quand on perçoit non des objets mais des êtres, qui sont dotés (entre autres) de caractéristiques sociales, c'est-à-dire ces éléments qui ne sont pas directement visibles à l'œil nu pour la plupart? Cela pose des questions spécifiques que l'auteur n'aborde pas directement, mais ses positions devraient aider à renouveler le questionnaire à ce propos. Si, comme le montre brillamment Benoist, nos concepts sont bien ceux d'êtres qui perçoivent, il doit en être de même pour nos concepts sociaux. Dans quelle mesure "voir un pauvre» (ou un riche) peut-il être le résultat d'une expérience perceptuelle? On pourrait croire les caractéristiques de race plus «naturellement " perceptuelles, mais «voir un noir» (ou un blanc) nous place au milieu d'une distinction qui n'a rien de perceptuel a priori, à savoir la division des êtres humains selon leur couleur de peau. Est-ce la même chose que de différencier les voitures selon leur couleur de peinture? C'est ce type de questions que l'auteur renvoie du côté d'une « microsociologie de la perception » (p.117), tout en les suggérant à partir de certains développements, comme ceux sur «voir un homme né à Jérusalem »; cette même microsociologie souvent invoquée mais qui n'a guère évolué ces dernières années.

17 Pour aller plus loin : qu'est-ce que cet arrangement perceptif qui consiste à «ne pas voir un noir », selon le fameux passage de Ralph Ellison dans L'homme invisible, à partir duquel Axel Honneth a fondé sa théorie de la reconnaissance (Honneth, 2005) ? Si la perception est bien ce fait dont on ne peut guère se défaire si ce n'est dans la mort (p. 9), alors la seule perte possible (de ce qui n'est donc pas une capacité) serait du côté d'une privation sensorielle (même si des altérations sont possibles, parfois recherchées: les oreillettes musicales, mais aussi l'alcool et autres drogues). A cela, il faudrait donc ajouter la conversion idéologique qui consiste à réellement ne plus vouloir voir les noirs, les homosexuels, etc. ${ }^{5}$

Les discussions de Benoist ont également le grand mérite de remettre au goût du jour certains passages déterminants d'Austin sur la perception, comme celui-ci : « 'J'ai vu un homme insignifiant en pantalon noir', 'j'ai vu Hitler' » (Austin, 2007, p. 189). Pour Austin, ces deux occurrences de "voir» ne dessinent pas des sens différents de ce verbe, cela montre plutôt qu'il n'existe pas de manière correcte de voir, et que sont réalisés à partir des perceptions des descriptions, des classements, des catégorisations qui diffèrent d'un individu à l'autre, en fonction de ses expériences précédentes, de sa socialisation, et de la situation présente dans laquelle il se trouve embarqué. Nous continuons à voir la même chose (le même individu, voire la même configuration spatiale ou le même environnement construit), mais le fait que nous soyons deux êtres sociaux différents nous la font voir plutôt comme ceci ou plutôt comme cela. C'est la manière qu'a Austin de relire le «voir... comme» wittgensteinien : les perceptions peuvent être partagées sans cesser d'être différenciées d'un individu à l'autre, du fait qu'elles sont nécessairement socialisées.

«Lorsqu'on voit quelque chose, il peut non seulement y avoir différentes façons de dire ce qu'on voit, mais la chose peut aussi être vue de différentes façons, être vue 
diversement. (...) Ainsi, les différences dans la manière de décrire ce qui est vu proviennent-elles très souvent, non pas simplement de différences dans notre savoir, dans la finesse de nos facultés discriminantes, dans notre propension à nous exposer, ou dans notre intérêt pour tel ou tel aspect de la situation totale; elles peuvent aussi provenir du fait que ce qui est vu est vu différemment, vu comme ceci plutôt que comme cela » (Austin, 2007, p. 191)

Cette approche distinguant partage et différenciation des perceptions, et plus largement ces perspectives, finement poursuivies par Benoist, dessinent une autre tradition sur la perception que son approche représentationnelle (cognitiviste) habituelle. Largement minoritaires dans les recherches actuelles, elles sont pourtant susceptibles de donner une assise théorique renouvelée aux travaux sur les ambiances en chamboulant la manière de comprendre tant la perception que le sensible, mais aussi de susciter de nouveaux questionnements du fait de ces prémisses remodelées.

Un dernier exemple de piste possible serait l'élargissement des déterminations phénoménologiques par la mise en évidence de l'origine perceptive de nombre de nos concepts ordinaires. Pour les travaux sur les ambiances, cela orienterait tant - pour les perspectives que l'on dira rapidement " qualitatives »- du côté d'une attention accrue à ces concepts ordinaires, à la façon dont ils entrent dans les descriptions ordinaires d'ambiances (dont ils les déterminent, les enrichissent, etc.); que - pour les perspectives que l'on dira tout aussi rapidement "quantitatives" - du côté de l'utilisation de nouveaux critères pour les mesures d'ambiances, à savoir des critères non plus seulement «techniques », mais aussi potentiellement fondés sur ces concepts ordinaires.

\section{BIBLIOGRAPHIE}

Al-Saleh, Christophe. 2004. L'usage des sens. Revue de métaphysique et de morale. $\mathrm{n}^{\circ}$ 42, p. 193-215.

Austin, John Langshaw. 2007 [1971]. Le Langage de la perception [trad.fr. par Gochet Paul, revue et introduite par Ambroise Bruno \& Laugier Sandra]. Paris : Vrin.

1994. Autrui. In : Écrits philosophiques [trad.fr. par Aubert Lou \& Hacker Anne-Lise]. Paris : Seuil.

Benoist, Jocelyn. 2009. Sens et sensibilité. L'intentionnalité en contexte. Paris : Le cerf.

Bentouhami, Hourya. 2014. L'emprise du corps. Fanon à l'aune de la phénoménologie de Honneth, Axel. 2005. Invisibilité : sur l'épistémologie de la « reconnaissance ». Réseaux. n 129-130, p. 39-57.

Merleau-Ponty. Cahiers philosophiques. n 138, p. 34-46.

Merleau-Ponty, Maurice. 1964. Le visible et l'invisible. Paris : Gallimard.

Pecqueux, Anthony. 2012. Les affordances des événements : des sons aux événements urbains. Communications. $\mathrm{n}^{\circ}$ 90, p. 215-227.

Pecqueux, Anthony. 2013. John Langshaw Austin, la perception et son ethnographie. In : Colon Paul-Louis (éd.). Ethnographier les sens. Paris : Petra. p. 43-70. 
Travis, Charles. 2004. The silence of the senses. Mind. vol. $113 \mathrm{n}^{\circ} 443$, p. 57-94 [version revue en 2010 disponible sur : http://mlag.up.pt/wp-content/uploads/2011/09/NewSilence.pdf, consultée le 15 octobre 2011].

\section{NOTES}

1. Citons au moins le précédent, Sens et sensibilité (Benoist, 2009), tout aussi décisif que celui dont il est question ici.

2. Senses are dumb, selon l'axiome de John Austin dans Le langage de la perception (2007) ou dans «Autrui » (1994, p. 70-71): «Les données sensorielles sont muettes, et seule notre expérience passée nous permet de les identifier ». Pour Austin, cet argument permet de récuser une théorie dominante à son époque, la théorie des sense-data : ces données sensorielles qui seraient issues des facultés sensorielles, en quelque sorte « dites » par ces dernières (actuellement, la cible d'une perspective austinienne serait plutôt la théorie représentationnelle de la perception). Si les sens sont muets, ils ne nous « disent» rien, ni indice, ni preuve de quoi que ce soit. Voir un cochon n'est pas un indice ou une preuve de sa présence ; je peux déceler, prélever progressivement des indices (olfactifs, auditifs, voire visuels : des traces dans la boue) de sa présence à proximité, mais une fois que je le vois, je le vois et c'est tout. Voir pour une exploration fine de cet argument philosophique majeur, Travis, 2004 et Al-Saleh, 2004 ; et pour ses conséquences ethnographiques, Pecqueux, 2013.

3. Ce passage est l'occasion de revisites riches de très belles pages du dernier Merleau-Ponty, celui du visible et l'invisible (1964), telle cette citation sur le sensible comme "possibilité d'être évident en silence, d'être sous-entendu » (p. 179) ; mais aussi des citations parfois énigmatiques, comme cette longue digression sur «l'objet en fil de fer dont je ne saurais dire ce qu'il est, ni combien de côtés il a, etc., et qui pourtant est là » (p. 180sqq), qui permet à Benoist de mettre en évidence l'indétermination ou plutôt la «dimension infra-significationnelle » de l'expérience perceptive (p. 183), qui est peuplée de choses qui sont à peine des objets.

4. Il peut être tentant de lire à cette aune le chapitre qui précède sur «les surprises de la perception ", donc sur les événements qui surgissent dans la perception et rendent manifestes par là des formes d'activités ; c'est du moins en ce sens que j'ai par ailleurs cherché à relire la notion d'affordance (Pecqueux, 2012).

5. Le récent essai d'Hourya Bentouhami (2014) offre de nombreuses pistes à ce propos en revisitant la lecture (tout à la fois implicite et explicite) de Merleau-Ponty par Frantz Fanon et en montrant ainsi tout l'apport pour la phénoménologie d'une analyse de la visibilité attentive au contexte politique.

\section{AUTEURS}

\section{ANTHONY PECQUEUX}

Chargé de Recherche au CNRS, CRESSON (UMR 1563 / Ecole Nationale Supérieure d'Architecture de Grenoble). Ses recherches portent de manière générale sur une approche écologique, sensible des expériences urbaines. Il a récemment dirigé le numéro 90 de la revue Communications, «Les bruits de la ville » (2012) ; et publié « John Langshaw Austin, la perception et son ethnographie » 
dans Ethnographier les sens (Paul-Louis Colon éd., 2013, Paris, Petra, p. 43-70), ou « Tordre

l'attention. Ajustements perceptifs en situation » dans L'économie de l'attention. Nouvel horizon du capitalisme? (Yves Citton éd., 2014, Paris, La Découverte, p. 219-228).

Il est membre du Comité Editorial de la Revue Ambiances.

Page personnelle : http://www.cresson.archi.fr/EQ/EQanthony.htm

Mail : anthony.pecqueux@grenoble.archi.fr 